PCOM). The most common microcatheters used were Headway Duo (10 cases), SL-10 (6 cases), Marksmann (4 cases) and Echelon 10 (4 cases). In the AIS cases, 69.2\% (9/13) achieved TICI $2 b-3$ recanalization. Ninety-six percent $(23 / 24)$ of IAs achieved successful obliteration Raymond-Roy Occlusion Classification grades (RROC I-II). All AVMs and dAVFs achieved complete embolization. Two procedural complications were reported: one temporal arterial occlusion in a patient with IA, and one permanent arterial thrombosis in a patient with AIS. Of 21 IAs with radiological follow-up, complete angiographic obliteration (RROC I-II) was observed in 95.2\% (20 cases).

Conclusions In this multicenter case series, the endovascular transcirculation approach was feasible and safe. Development of newer endovascular devices will further improve angiographic results and neurological outcomes in these complex cases.

Disclosures J. Roa: None. G. Dabus: None. A. Maud: None. M. Martinez-Galdamez: None. D. Paez-Granda: None. V. Kalousek: None. A. Mowla: None. V. Szeder: None. P. Jabbour: None. S. Ortega-Gutierrez: None. D. Hasan: None. E. Samaniego: None.

\section{E-019 COMPARE THE CLINICAL RESULTS BETWEEN THE CLIPPING \& COILING IN RUPTURED INTRACRANIAL ANEURYSM PATIENTS}

${ }^{1} Y$ Won*, ${ }^{2}$ D Yoo. ' Radiology, Uijeongbu St. Mary's Hospital, Uijeongbu-si, Korea, republic of; ${ }^{2}$ Neurosurgery, Eunpyeong St. Mary's Hospital, Seoul, Korea, republic of

\subsection{6/neurintsurg-2019-SNIS.94}

Background and purpose Craniotomy with neck clipping or endovascular coiling is treatment modality for cerebral aneurysm. In general, coiling is faster and less invasive, but not able to remove the subarachnoid hemorrhage. It is well known that substances released from the blood from the subarachnoid hemorrhage and inflammatory process might be the main cause of vasospasm and hydrocephalus. Authors analyzed our data to compare the incidence of vasospasm and hydrocephalus according to treatment modalities.

Methods Total 1039 cerebral aneurysm patents (723 ruptured \& 315 unruptured; among 723 ruptured cases, 320 patients clipped \& 403 patients coiled) between March 2007 \&amp February 2017, were include in this analysis. In this study, most MCA aneurysms were treated clipping while most posterior circulation aneurysms were treated coiling. Authors compare the incidence of vasospasm and chronic hydrocephalus, procedure time, neurologic improvement rate and re-treatment rate.

Results Vasospasm developed $10.6 \%$ in clipping and $5.5 \%$ in coiling group $(p=0.007)$. Chronic hydrocephalus, need shunt operation, developed similar incidence in both groups $(11.6 \%$ in clipping and $9.4 \%$ in coiling, $p=0.208)$. The procedure time (from anesthesia induction to wake up time) was 114.8 minutes in coiling while it was 394.2 minutes in clipping group $(p=0.000)$. Initial neurologic status was worse, but neurologic improvement (= eGCS - iGCS) was frequent in coiling group $(57.6 \%$ in coiling and $40.9 \%$ in clipping, $\mathrm{p}=0.000)$. Re-treatment rate $(p=0.238)$ were statistically no difference in both groups.

Conclusions From our study, mechanical irritation to the offending vessel might be the most important cause of vasospasm development while subarachnoid hemorrhage does not influence on the hydrocephalus development. Overall retreatment rate after coiling and clipping was similar.

Disclosures Y. Won: None. D. Yoo: None.

\section{E-020 SINGLE CENTER ANALYSIS OF EFFICACY OF HYDROGEL COILS IN TREATMENT OF ANTERIOR COMMUNICATING ARTERY ANEURYSMS}

${ }^{1} \mathrm{G}$ Malaty*, ${ }^{2} \mathrm{~B}$ Patel. 'Virginia Tech Carilion School of Medicine, Roanoke, VA; ${ }^{2}$ Neurointerventional Surgery, Department of Radiology, Carilion Clinic, Roanoke, VA

\subsection{6/neurintsurg-2019-SNIS.95}

Introduction/Purpose Approximately 30-37\% of all cerebral aneurysms occur in the anterior communicating artery (AComm), making it the most common site for such lesions. These aneurysms constitute $12-15 \%$ of all unruptured and $23-40 \%$ of all ruptured cerebral aneurysms, and account for approximately $40 \%$ of aneurysmal subarachnoid hemorrhage (SAH) in adults. ${ }^{1,2}$ AComm aneurysms, when compared to other anterior circulation cerebral aneurysms, are more likely to rupture, as 50\% of those that experience rupture do so at less than $7 \mathrm{~mm}$ diameter ${ }^{2}$. Series outlining the safety and efficacy of hydrogel coils in aneurysm treatment show favorable angiographic outcomes, reduced thromboembolic complications, reduced recanalization, recurrence, and procedure-related morbidity and mortality when compared to bare platinum coils. ${ }^{3,4}$ However, despite the demonstrated success of hydrogel coils in treating cerebral aneurysms at large, no studies in the United States to date have examined outcomes related to the use of these coils versus bare platinum coils in the treatment of aneurysms of the anterior communicating artery, specifically. This study aims to compare these coil types for efficacy in terms of aneurysm obliteration and recurrence, and coil number and packing density.

Materials and methods A retrospective chart review of 36 patients with untreated, ruptured and unruptured, saccular AComm aneurysms who were treated via bare platinum or hydrogel-coated coil embolization during the period of August 2014 to present was conducted. Data extracted from patient charts included aneurysm size, morphology, rupture status, endovascular coil number, type, and size. Additionally, each aneurysm was assigned a Raymond-Roy Occlusion Classification (RROC) grade based on accompanied followup imaging at 6-12 months. RStudio was used to conduct all relevant statistical analysis including Welch's two-sample t-testing, least-squares regression, and non-parametric testing.

Results Hydrogel coil-treated AComm aneurysms, when compared to those treated with bare metal coils, demonstrated an equal rate of aneurysm obliteration with reductions in average number of coils used per aneurysm $(\beta=$ $0.4150, \mathrm{P}=0.027)$, packing density per aneurysm $(\mathrm{t}(26.1)$ $=2.09, \quad 95 \%$ CI $0.19-22.62, \quad \mathrm{P}=0.046)$, retreatment $(\mathrm{P}=0.031)$, and median six-month recurrence $(\mathrm{W}=183$, $\mathrm{P}=0.043$ ).

Conclusions In the setting of coil embolization of AComm aneurysms, hydrogel-coated endovascular coils exhibit similar rates of aneurysm obliteration when compared to bare platinum coils, but achieve these obliteration rates with lower mean packing densities, fewer coils used, and lower 
overall recurrence and retreatment. Based on these findings, we conclude hydrogel coils to be more efficacious than their bare metal counterparts in treating AComm aneurysms.

\section{REFERENCES}

1. Cai, Wu, et al. 'Anterior communicating artery aneurysm morphology and the risk of rupture.' World neurosurgery 109 (2018): 119-126.

2. Bijlenga, Philippe, et al. 'Risk of rupture of small anterior communicating artery aneurysms is similar to posterior circulation aneurysms.' Stroke 44.11 (2013): 3018-3026.

3. Park $\mathrm{JH}$, et al. 'Embolization of intracranial aneurysms with HydroSoft coils: results of the Korean multicenter study.' American Journal of Neuroradiology 32.9 (2011): 1756-1761.

4. White, Philip $M$, et al. 'Hydrogel-coated coils versus bare platinum coils for the endovascular treatment of intracranial aneurysms (HELPS): a randomised controlled trial.' The Lancet 377.9778 (2011): 1655-1662.

Disclosures G. Malaty: None. B. Patel: 1; C; MicroVention, Inc.

\section{E-021 IN VITRO NEUROVASCULAR MODEL DEVELOPMENT FOR LIQUID EMBOLIC IMPLANT SIMULATION}

C Settanni* ${ }^{*}$ T Becker, A Ducruet, W Merritt, A Huckleberry. Bioengineering Devices Lab, Mechanical Engineering, Northern Arizona University, Flagstaff, $A Z$

10.1136/neurintsurg-2019-SNIS.96

Introduction Although current microcatheter technologies have advanced in recent years, corresponding endovascular devices still lag behind. Animal models are unable to replicate consistent large and wide neck bifurcation aneurysms with sufficient neurovascular feeder vessels. Testing methods must accurately model vessel tortuosity and flow patterns. Models must detect downstream migration of neurovascular embolic devices. This paper focuses on the development and utilization of an in vitro flow model to test short- and long-term stability of a novel polymer biomaterial (PPODA-QT) for aneurysm occlusion.

Materials and methods This research project includes development of innovative in vitro aneurysm vessel model with complex side branching. This project will bring together clinical, biological, and engineering expertise. A full Circle of Willis (CW) in vitro vessel model will be fabricated into vessel analogs. This model will be constructed using a PolyJet (UV cured) $3 \mathrm{D}$ printing process. UV curing creates a model with accurate anatomy and tortuosity. Typical aneurysm positions, verified by our collaborating neuro-interventional surgeon, will be $3 \mathrm{D}$-printed at the basilar bifurcation, the posterior communicating (PCA) branch, and at the anterior communicating (ACA) bifurcation. Flow is regulated through the use of a Super Pump AR (ViVitro Labs). This 3D printed model will be implemented in an in vitro flow model that enables systemic data collection. A data acquisition system (DAQ) integrated with LabVIEW $^{\circledast}$ software will record real time particulation images, pressure drops across the model, and flowrates through each inlet and outlet of the model.

Results and discussion The in vitro model is used to simulate and quantify short-and long-term viability of new biomaterials for brain aneurysm embolization. Delivery and balloon microcatheters access the aneurysm model from the introducer at the inlet flow-stream. Inline holography imaging quantifies the number and size of particulate in accordance with (USP) $\mathrm{XXV}<788>$ ). The PolyJet $3 \mathrm{D}$ printed model has a luminal friction $5 \mathrm{x}$ lower than the standard silicone vessel models.
Low luminal friction improves endovascular device tracking, providing a realistic feel for surgical simulation.

Conclusions This in vitro aneurysm flow model utilizes a UV cured 3D printing technique to emulate device delivery and wear. This model provides a realistic simulation of neurovascular device delivery. Additionally, development of new biomaterials for aneurysm treatment requires models capable of replicating tortuosity and friction characteristics. Traditional additives (used to reduce wall friction) can interfere with deployment and assessment of embolics.

Disclosures C. Settanni: None. T. Becker: 1; C; Brain Aneurysm Foundation. 4; C; Aneuvas Technologies Inc. 5; C; Aneuvas Technologies Inc., Northern Arizona University. A. Ducruet: 1; C; Brain Aneurysm Foundation. 5; C; Barrow Neurological Institute, Northern Arizona University. W. Merritt:None. A. Huckleberry: 5; C; Flagstaff Medical Center.

\section{E-022 SEX DIFFERENCES IN ANEURYSM MORPHOLOGY AND LOCATION}

${ }^{1} \mathrm{E}$ Roney ${ }^{*},{ }^{2} \mathrm{D}$ Lukins, ${ }^{1} \mathrm{M}$ Nisiewicz, ${ }^{2} \mathrm{~A}$ Alhajeri, ${ }^{3} \mathrm{~S}$ Grupke, ${ }^{3} \mathrm{~J}$ Fraser. ${ }^{1}$ University of Kentucky College of Medicine, Lexington, $K Y_{;}{ }^{2}$ Department of Radiology, University of Kentucky, Lexington, $\mathrm{KY}$; ${ }^{3}$ Department of Neurosurgery, University of Kentucky, Lexington, KY

10.1136/neurintsurg-2019-SNIS.97

Background and Purpose Risk factors for formation and rupture of intracranial aneurysms (IAs) have been extensively investigated, and it is apparent that IAs and subarachnoid hemorrhage (SAH) are more common in females than males. Much is unknown about why IAs occur more frequently in females, but previous investigators have implicated hormonal and flow geometry variations in the development of compromised vessel wall integrity. The purpose of this study was to evaluate morphological characteristics of aneurysms within our population and differences in morphology and/or anatomic locations between males and females.

Methods A retrospective review was performed of patients undergoing 3D angiograms for intracranial aneurysms at a comprehensive stroke center between January 1, 2012 and January 1, 2018. Data collected included patient demographic information, comorbidities and aneurysm characteristics evaluated by $3 \mathrm{D}$ angiogram. All statistical analyses were completed in SAS 9.4.

Results 276 patients (203 female, 73 male) with 404 aneurysms were included. Of the 276 patients, 113 (81 female, 32 male) presented with ruptured IAs. On univariate analysis, female patients had significantly greater odds of having a posterior circulation aneurysm than males $(p=0.0073$; OR, $4.30 ; 95 \%$ CI 1.48 to 12.48$)$. For males and females, the most frequent locations were the anterior communicating artery and internal carotid artery, respectively. No statistically significant differences were observed in neck diameter, dome to neck ratio, or presence of secondary aneurysmal sacs.

Conclusion The sex differences in aneurysm morphology and location require further investigation. Aneurysms found in male and female patients had different anatomic distributions; the most frequent location was the anterior communicating artery for men and the internal carotid artery for women. No statistically significant differences were discovered in the studied morphological parameters. Sex differences in IA characteristics may reveal unknown information about the pathophysiology of formation of IAs and possible disparities in 